\title{
Methods to visualize auxin and cytokinins signalling activity in the shoot apical meristem
}

Géraldine Brunoud, Carlos S. Galvan-Ampudia and Teva Vernoux*

Laboratoire Reproduction et Développement des Plantes (RDP), Univ Lyon, ENS de Lyon, UCB Lyon 1, CNRS, INRA, F-69342, Lyon, France

*For correspondence: teva.vernoux@ens-lyon.fr

\begin{abstract}
Visualizing the distribution of hormone signalling activity such as auxin and cytokinins is of key importance for understanding regulation of plant development and physiology. Live imaging and genetically encoded hormone biosensors and reporters allow monitoring the spatial and temporal distribution of these phytohormones. Here, we describe how to cultivate live shoot apical meristems after dissection for observation under the confocal microscope for up to four days. The shoot apical meristems are maintained on an appropriate medium allowing them to grow and initiate new organs at a frequency similar to plants grown on soil. Meristems expressing hormone biosensors and reporters allows following hormone signalling activity distribution at high spatio-temporal resolution without chemical fixation, an approach that that can also be applied to follow the dynamics of expression in vivo of any fluorescent marker.
\end{abstract}

Keys words: shoot apical meristem, phytohormones biosensors and reporters, live imaging, confocal microscopy.

\section{INTRODUCTION}

The plant hormones auxin and cytokinins are key regulators of growth and development. Both are small molecules, tryptophan-derived and adenine-derived respectively, which are very difficult to detect in vivo at high spatio-temporal resolution. The shoot apical meristem (SAM) is a specialized tissue located at the tip of the growing stem in which these hormones plays a key role. The SAM contains a stem cell niche and produces most of the above ground parts of plants, including stem, leaves, shoots and flowers. In the SAM (as in other parts of the plant), auxin is a central regulator of patterning and organogenesis. It is actively and polarly transported and this transport creates auxin differential distributions or gradients throughout the tissue [1]. This non-homogenous distribution in the tissue is notably essential to organ initiation that is triggered by local accumulation of auxin. Cytokinins have been shown to promote stem cell identity and maintenance in the central zone of the 
meristem [2]. They are also regulating the timing of organ initiation at the shoot apical meristem and as a result phyllotaxis robustness [3].

Several sensors and reporters for auxin and cytokinin are currently available. The auxin signalling input can be visualised using the DII-VENUS degradation-based biosensor, or its R2D2 derivative [4, 5], that has been engineered using the degron of an Aux/IAA protein fused to the fast maturating VENUS yellow fluorescent protein and a nuclear localization signal (NLS). Aux/IAA are degraded upon perception of auxin and DII-VENUS fluorescence thus provides information on the combined action of auxin concentration and of auxin perception capacity in plant cells. AUXIN RESPONSE FACTORs (ARFs) and ARABIDOPSIS RESPONSE REGULATORs (ARRs) are transcriptional factors that activate gene expression in response to auxin and cytokinin respectively. Synthetic reporters consisting in multiple ARF or ARR binding sites fused to a minimal promoter and driving a reporter protein allows following the transcriptional activity induced downstream of auxin and cytokinin (signalling output) respectively: auxin-induced transcription can be followed using the DR5 synthetic promoter [6, 7] or the DR5v2 promoter [5] while the TCS and TCSn synthetic promoter allows following cytokinin-induced transcription $[8,9]$. These different genetically encoded markers have been largely used to follow auxin distribution and cytokinin signalling at the shoot apical patterning [3, 4, 10-12].

Live imaging microscopy of genetically encoded fluorescent reporters allow following the spatiotemporal dynamics of hormone distribution at the shoot apical meristem during developmental and physiological responses [11-13]. Here we provide a detailed description of how to cultivate live shoot apical meristems after dissection for observation under the confocal microscope for up to four days. We show how using the spatio-temporal distribution of auxin and cytokinin activity can be followed with hormone biosensors and reporters and correlated with key developmental processes such as stem cell maintenance, organogenesis and patterning. 


\section{BIOLOGICAL MATERIAL}

As introduced above, we use Arabidopsis plants expressing markers constructed using DR5, DR5vé and TCS promoters to follow hormone-induced transcription. We use here DR5::3xVENUS-N7 and DR5V2::ntdTomato $[5,11]$. The two reporters differ not only in the fluorescent protein (FP) driven by the promoter but also in the repetitive DNA sequences bound by ARF (Auxin Responsive Elements -AuxRE-) in the promoter [5]. DR5v2 has been demonstrated to have a higher sensitivity than DR5 in certain tissues. For Cytokinin, we use the TCS::GFP reporter [8].

To detect rapid changes in auxin concentration and signalling activity, we use the degradation-based biosensors DII-VENUS and R2D2 [4, 5]. DII-VENUS is driven by the $35 S$ ubiquitous promoter and R2D2 consists in a single construct with DII-VENUS and $m D I I-$ $n t d T o m a t o$ driven by two independent RPS5a promoters that provide a strong and ubiquitous expression in the SAM. While the DII sequence allows for a rapid degradation by auxin, this degradation is strongly inhibited by the mutation present in the $m D / l$ sequence. $R 2 D 2$ thus provides a ratiometric reading of the auxin-dependent degradation of DII-VENUS.

All the Arabidopsis lines used here are in the Col-0 ecotype except R2D2 that is in the Columbia-Utrecht (Col-utr) ecotype [14].

\section{MATERIALS}

\section{1-Half-MS Medium}

Prepare medium with:

- Half MS medium without vitamins,

- Sucrose $1 \%$

Adjust $\mathrm{pH}$ to 5,8 with $1 \mathrm{M} \mathrm{KOH}$

Add Agarose at $0.8 \%$ final

Autoclave the medium

Use to pour plates or store at RT once solidified until plates are needed

\section{$\underline{\text { 2-Vitamins stock (1000X) }}$}

Myo-inositol

Nicotinic acid

Pyrodoxine hydrocholorid

Thiamine hydrochloride

Glycine

Add Water
$5 g$

$0.05 \mathrm{~g}$

$0.05 \mathrm{~g}$

$0.5 \mathrm{~g}$

$0.1 \mathrm{~g}$

$50 \mathrm{ml}$ 
Sterilize using $22 \mu \mathrm{m}$ filtration filters, aliquot and store at $-20^{\circ} \mathrm{C}$ and use for no longer than a year.

The aliquots can be frozen and defrozen several times.

\section{3- N6-Benzyladenine (BAP, a cytokinin)}

Prepare a $100 \mu \mathrm{M}$ stock solution in water.

To allow for the complete dissolution, add $1 \% \mathrm{v} / \mathrm{v}$ of $\mathrm{NaOH} 1 \mathrm{~N}$.

Sterilize using $22 \mu \mathrm{m}$ filtration filters, store aliquots at $-20^{\circ} \mathrm{C}$ and use for up to six months.

Once defrozen, the aliquots should be discarded after use.

\section{$\underline{4-D y e s ~ t o ~ s t a i n ~ c e l l s ~ c o n t o u r s ~}$}

-Plasma membrane staining using FM4-64 (N-(3-Triethylammoniumpropyl)-4-(6-(4-(Diethylamino) Phenyl) Hexatrienyl) Pyridinium Dibromide)

Prepare a stock solution at $1 \mathrm{mg} / \mathrm{ml}$ in sterile water. Store aliquots at $-20^{\circ} \mathrm{C}$ and use for no longer than a year.

Once defrozen, the aliquots should be put back to $-20^{\circ} \mathrm{C}$ only once for maximal staining.

-Cell wall staining using Propidium lodide (PI, 3,8-Diamino-5-[3-(diethylmethylammonio)propyl]-6phenylphenanthridinium diiodide)

Use directly solution from Sigma Aldrich available at $1 \mathrm{mg} / \mathrm{ml}$ (Ref: P4864). Store in fridge at $+4^{\circ} \mathrm{C}$.

\section{5-Tools for dissection}

Forceps: Size $N^{\circ} 5$ Roth (ref: LH79.1)

Petri dishes: 60X10mm Falcon (ref: 353004)

Porous tape

White and soft facial tissue with high absorption

\section{$\underline{\text { 6-Microscope equipment }}$}

-Stereomicroscope

-Confocal microscope upright (Zeiss LSM700) equipped with a long distance objective X40 water immersion (for example we use a Zeiss W "N-Achroplan" 40x/0.75 M27 (ref 420967-9900), water, working distance $2.1 \mathrm{~mm}$, without cover glass). 
-Confocal microscope inverted (Zeiss LSM710) equipped with a long distance objective X40 water immersion (for example we use a Zeiss W "N-Achroplan" 40x/1.0 M27 (ref 421462-9900), water, working distance $2.5 \mathrm{~mm}$, without cover glass). 


\section{METHODS (Fig1)}

\section{1-Preparation of the in vitro Apex Culture Medium (ACM) plates}

Melt the half-MS medium.

Add vitamins (final concentration 1X) and BAP (final concentration 200nM) under a laminar hood, sterile conditions (see note1). Pour the medium in Petri dishes.

Use directly or store at $+4^{\circ} \mathrm{C}$ for no longer than a month.

\section{2-Plant growth conditions}

The following growth conditions are required:

- Short day conditions: 8 hours light and 16 hours night, $21^{\circ} \mathrm{C}$, hygrometry $55-60 \%$.

- Long day conditions: 16 hours light at $21^{\circ} \mathrm{C}$ and 8 hours night at $18^{\circ} \mathrm{C}$, hygrometry $55-60 \%$

To obtain healthy plant and facilitate dissection, plants are germinated in short day condition and grown in these conditions for 4 weeks. Plants are transferred for two weeks in long day conditions to induce flowering and development of the inflorescence (optimal conditions, Fig1A). Water the plants at least two hours before dissection. It is possible to dissect meristems from plants grown in long days only or secondary and axillary meristems but the smaller size of the stem makes the dissection more difficult.

\section{$\underline{\text { 3-Dissection (Fig1B, C, D, E) }}$}

Dissect from 6 to $24 \mathrm{~h}$ before the first observation (see, note 2 ).

First by naked eye, remove as much as possible of the older flowers with the forceps (Fig1B). Then using the stereomicroscope, there are two alternative ways to continue with the dissection depending on the length of the inflorescence stem. Meristems with stems from 5 to $30 \mathrm{~mm}$ long can be dissected directly on the plant (Fig1C). Inflorescences longer than $30 \mathrm{~mm}$ can be cut and dissect on medium plate, by cutting at $1 \mathrm{~cm}$ for the top and transferring the segment of stem onto the medium plate (Fig1D). Then remove old primordia one by one using forceps, try to cut the petioles more closely to stem as possible. The meristem appears at the centre as a green dark tissue in comparison with the primordia (Fig2), (see note 3).

A last methods for very long stem and old plant: cut the stem at their based and placed horizontally on a piece of craft paper and dissected by rolling the stem with the fingers (Fig1E) and remove all lateral organs progressively using forceps (see note 4).

After dissection, transfer the meristems to clean medium plates (see note 5 and note 6 ) that will be used for microscope acquisition (Fig1F), taking care of aligning the stem with the gravity axis. Close the plates with porous tape and place the dishes back in the culture chamber or in a growth incubator with similar growth conditions. 


\section{5-Before microscopy}

Use a stereo microscope to check the dissected SAM as the sample might require additional dissection. Again, it is also important to check that the meristem is vertically aligned (Fig1F and Fig2).

Submerge the meristem with sterile water for 8 minutes by filling the dish (see note 7).

\section{6-Staining of the cell contours (optional)}

Stain meristems with PI (at $100 \mu \mathrm{M}$ final concentration in water) for 5 minutes (see note 8). In order to reduce staining volumes, we place a sterile $1000 \mu \mathrm{L}$ tips (cutted about $2 \mathrm{~cm}$ from the top of the tip) around the dissected sample and add sufficient PI solution (about $150 \mu \mathrm{l}$ ) to fully cover the meristems (Fig 1G). After incubation, rinse twice with sterile water (Fig1H), (see note 9).

Alternatively SAMs can be stained with FM4-64. Add FM4-64 (at $300 \mu \mathrm{M}$ final concentration in water) for 10 minutes by applying $10 \mu$ (one drop) on top of the dissected meristem. The drop of FM4-64 solution should stay stably on the SAM for optimal staining. Rinse briefly twice with sterile water.

\section{7-Acquisition}

With an upright microscope (Fig1I), place the imaging dish onto the stage, then lower the microscope lens until it touch the surface of the water (see note 10), be careful to avoid air bubbles under the lens.

With an inverted microscope (Fig1J), apply a drop of sterile water on the lens, hold the imaging dish upside down on the stage then raise the microscope lens until a water column is formed between the lens and medium(see note 10 and note 11 ).

Using transmitted light, position the sample within field of the objective, then focus on the top of the meristem using the $Z$ control. It is also possible to use the epifluorescence illumination to find the meristem, however this might lead to fluorescence bleaching of the sample and should be avoided if not absolutely necessary.

Adjust the confocal settings (laser power, gain, offset) accordingly to the signal. To avoid signal saturation, use the range indicator palette, and set the gain until there is no or few red points in the image. It is recommended to use a minimal laser power to avoid photo-bleaching. It is also possible to open the pinhole up to 1.5 or 2 . We use the following settings: for VENUS (DR5, DII and R2D2) laser 514nm (5\% power) and emission range: $520-560 \mathrm{~nm}$, for ntdTomato (R2D2) laser $561 \mathrm{~nm}(5 \%$ power) and emission range: $580 \mathrm{~nm}-620 \mathrm{~nm}$ and GFP (TCS) laser 488 (5\% power) and emission range $467-537 \mathrm{~nm}$. For $\mathrm{PI}$ and $\mathrm{FM} 4-64$ we used the $488 \mathrm{~nm}$ laser at $5 \%$ power and adjust the emission range from 646 to $660 \mathrm{~nm}$ to avoid auto fluorescence interference [15] (see note 12).

\section{8-After imaging}

When doing a time course experiment (see note 13 and note 14), it is important to remove the excess of water from the sample. Under the stereomicroscope, the meristem has to appear shiny and without any sign of water on it or on the medium before putting it back to grow. To remove all the 
water after imaging, use a clean absorbent facial tissue to absorb any drop on the medium and let the plate dry for 4 to 6 minutes before closing it. When necessary, remove older organs after image acquisition not before to avoid stressing the meristem (see note 15).

\section{9-Analysis/post processing}

For image processing and signal quantification, ImageJ or FiJi (http://imagej.net/Welcome or https://imagej.net/Fiji) offer multiple tools. It is out of our scope to describe all of them here and we are just giving here a few hints on how to analyse the data. Maximal intensity Z-projections help to visualise the distribution of the different markers. Figure 3. Show projections obtained using Fiji: in A; nuclear DR5 signal (LUT fire) and cell wall stained by PI (in yellow), in B; nuclear DII (LUT blue orange icb) and auto fluorescence (in red), in C; reticulum endoplasmic TCS signal (LUT Green Fire Blue) and auto fluorescence (in red), in D; R2D2 line with the DII signal (LUT Rainbow) and the constitutive expression (in green).

Further quantification and analysis of meristems can require tissue segmentation and 3D reconstruction. For this MorphoGraphX MGX (http://www.mpipz.mpg.de/MorphoGraphX/ P.), [16] or MARS-ALT (http://openalea.gforge.inria.fr/doc/vplants/vtissue/doc/ build/html/user/index.html [17] can be used. We refer the reader to the corresponding website and publications for use of these approaches

\section{NOTES}

1. Wait for medium temperature to reduce to $50-55^{\circ} \mathrm{C}$ to avoid denaturation of vitamins and BAP.

2. Let the meristem recover from the dissection. Mechanical or wounding stresses are to be avoided as they can affect the fluorescence distribution of the reporters. DII is particularly sensitive and dynamics, the DII signal completely disappears within minutes after the dissection but reappears progressively.

3. The dissection should be as rapid as possible to limit desiccation of the meristem.

4. The rolling method induces more mechanical stresses and a faster desiccation of the sample, but can be very useful when the plants have grown more rapidly than expected.

5. Let the plate at room temperature at least 30 minutes before using to avoid cold stress to the meristem.

6. Dissect a double number of meristems than the number you expect to image and analyse because some of them will not survive over a long time course.

7. It is crucial to immerge the sample in water few minutes before imaging to avoid sample movement during acquisition due to water absorption and swelling of the gel.

8. PI staining reveal damage, because when the tissue is damaged the PI will penetrate inside and the signal will be strong and/or in the nucleus rather than well localized at the cell wall.

9. The cut $1000 \mu \mathrm{L}$ tips can then be reused. They need then to be kept in Ethanol $70 \%$ to avoid contamination. 
10. Use a water immersion objective to avoid desiccation, if dry objective is used the imaging time should be less than one minute.

11. During long imaging time regularly check the water column and add water if necessary.

12 When imaging meristems with different fluorophores, we recommend sequential (multi-track) acquisition to avoid cross-channel signal contamination.

13. Bacteria or fungus contamination will kill the meristem. To avoid this use a new bottle of sterile water at each imaging time. The objective has also to be cleaned before imaging with $70 \%$ ethanol.

14. For long time courses (more than 2 days), the ACM medium has to be change (every other day) to avoid contamination but also be certain that the BAP concentration is stable.

15. A way to know the meristem is still alive and in good condition is that you should detect new organ initiation at around one new organ per 12 hours.

\section{REFERENCES:}

1. Petrasek J. and Friml J. (2009) Auxin transport routes in plant development. Development 136, 2675-2688

2. Pfeiffer A., Wenzl C. and Lohmann J. U. (2017) Beyond flexibility: controlling stem cells in an ever changing environment. Current Opinion in Plant Biology 35, 117-123

3. Besnard F., Rafahi Y., Morin V., Marteaux B., Brunoud G., Chambrier P., Rozier Frédérique, Mirabet V., Legrand J., Lainé S., Thévenon E., Farcote E., Cellier C., Das P., Bishopp A., Dumas R., Parcy F., Helariutta Y., Boudaoud A., Godin C., Traas J and Vernoux T (2013) Cytokinin signalling inhibitory fields provide robustness to phyllotaxis. Nature 550, 417-421

4. Brunoud G., Wells D. M., Oliva M., Larrieu A., Mirabet V., Beeckman T., Kepinski S., Traas J., Bennett M. J., and Vernoux T. (2012) A novel sensor to map auxin response and distribution at high spatio-temporal resolution. Nature 482, 103-106

5. Liao C., Smet W., Brunoud G., Yoshida S., Vernoux T. and Weijers D (2012) Reporters for sensitive and quantitative measurement of auxin response. Nature Methods 12, 207-210

6. Ulmasov T., Murfett J., Hagen G. and Guilfoyle T. J. (1997) Aux/IAA proteins repress expression of reporter genes containing natural and highly active synthetic auxin response elements. The Plant Cell 9, 1963-1971 
7. Sabatini S., Beis D., Wolkenfelt H., Murfett J., Guilfoyle T., Malamy J., Benfey P., Leyser O., Bechtold N. Weisbeek $P$ and Scheres B (1999) An auxin dependent distal organizer of pattern and polarity in the Arabidopsis root. Cell 99, 463-472

8. Muller B. and Sheen J. (2008) Cytokinin and auxin interaction in root stem-cell specification during early embryogenesis. Nature 453, 1094-1097

9. Zürcher E., Tavor-Deslex D., Lituiev D., Enkerli K., Tarr P. T. and Müller B. (2013) A robust and sensitive synthetic sensor to monitor the transcriptional output of the cytokinin signalling network in plant. Plant Physiology 161, 10066-1075

10. Benkova E., Michniewicz M., Sauer M., Teichmann T., Seifertova D., Jürgens G and Friml J (2003) Local, efflux-dependent auxin gradients as a common module for plant organ formation. Cell 115, 591-602

11. Heisler M. G., Ohno C., Das P., Sieber P, Reddy G. V., Long J. A. and Meyerowitz E. (2005) Patterns of auxin transport and gene expression during primordium development revealed by live imaging of the Arabidopsis inflorescence meristem. Current Biology 15, 1899-1911

12. Vernoux T., Brunoud G., Farcot E., Morin V., Van den Daele H., Legrand J., Oliva M., Das P., Larrieu A., Wells D., Guédon Y., Armitage L., Picard F., Guyomarc'h S., Cellier C., Parry G. Koumproglou R., Doonan J. H., Estelle M., Godin C., Kepinski S., Benette M., De Veylder L and Traas J (2011) The auxin signalling network translate dynamic input inti robust patterning at the shoot apex. Mol. Syst. Biol 7, 508

13. Adibi M., Yoshida S., Weijers D. and Fleck C. (2016) Centering the organizing center in the Arabidopsis thaliana shooy apical meristem by combination of cytokinin signalling and selforganization. PLOSone 11(2): e0147830

14. Liao C., Smet W., Brunoud G., Yoshida S., Vernoux T. and Weijers D (2015) Corrigendum: Reporters for sensitive and quantitative measurement of auxin response. Nature Methods 12, 1098

15. Chudakov D., M., Matz M. V., Lukyanov S. and Lukyanov K. A. (2010) Fluorescent proteins and their applications in imaging living cells and tissues. Physiol Rev 90, 1163-2010

16. Barbier de Reuille P., Robinson S. and Smith R. S. (2015) Quantification cell shape and gene expression in the shoot apical meristem using MorphographX. Plant Cell Morphogenesis: Methods and Protocols. Methods in Molecular Biology 1080, 121-134 
17. Fernandez R., Das P., Mirabet V., Moscardi E., Traas J., Verdeil J-L., Malanchain G. and Godin C. (2010) Imaging plant growth in 4D : robust tissue reconstruction and lineaging at cell resolution. Nature Methods 7, 547-553

\section{FIGURE LEGENDS}

Fig1: Illustration of the experimental procedure. A: optimal size of the plant for the dissection. B, C, $D$, E: several steps of the dissection procedure as described in the text. F: Meristems on an ACM plate at the end of the dissection. G: Set-up for PI staining. H: Wash after staining. I, J: Set-up for acquisition using upright $(\mathrm{I})$ and inverted $(\mathrm{J})$ microscope. $\mathrm{K}$, L: Visualization of fluorescence in a meristem on a Zeiss LSM710 inverted confocal microscope and Zen associated software.

Fig2: Visualization under the stereomicroscope of a dissected Shoot Apical Meristem on the ACM medium. Note the darker colour of the meristem at the center. Scale Bar: $50 \mu \mathrm{m}$

Fig3: Maximum projections performed using Fiji.

A: DR5::3VENUS-N7 expression in LUT fire, cell wall stained by PI in yellow. B: DII-VENUS in LUT blue orange icb, auto fluorescence in red. C: TCS::erGFP in LUT Green Fire Blue, auto fluorescence in red. D: R2D2; DII in LUT Rainbow, constitutive expression in green. Scale bar $50 \mu \mathrm{m}$ 